\title{
Retrospective Study
}

\section{e Transforaminal Endoscopic Discectomy with Foraminoplasty for the Treatment of Spondylolisthesis}

Gabriele P. Jasper, MD', Gina M. Francisco, MBS'1, and Albert E. Telfeian, MD, PhD²

From: ${ }^{1}$ Center for Pain Control, Brick, NJ; 'Brown University, Providence, RI

Address Correspondence: Albert E. Telfeian, MD, PhD Brown University Dept of Neurosurgery 593 Eddy Street Rhode Island Hospital Dept. of Neurosurgery Providence, RI E-mail:

albert.telfeian@nts-online.net

Disclaimer: There was no external funding in the preparation of this manuscript. Conflict of interest: Each author certifies that he or she, or a member of his or her immediate

family, has no commercial association (i.e., consultancies, stock ownership, equity interest, patent/licensing arrangements, etc.) that might pose a conflict of interest in connection with the submitted manuscript.

Manuscript received: 04-14-2014 Revised manuscript received: 06-28-2014 Accepted for publication: 07-11-2014

Free full manuscript: www.painphysicianjournal.com
Background: Lumbar degenerative spondylolisthesis is a common entity and occurs mainly in elderly patients. The trend in surgery has been to offer decompression with instrumented fusion based on patient-based outcome data and the inherent instability of the condition.

Objectives: Transforaminal endoscopic discectomy and foraminotomy is an ultra-minimally invasive outpatient surgical option available to patients that does not require general anesthesia and does not involve the same amount of destabilizing facet joint removal as a traditional laminectomy and medial facetectomy. The purpose of this study was to assess the benefit of tranforaminal endoscopic discectomy and foraminotomy in patients with lumbar 4-5 (L4-L5) and lumbar 5-sacral 1 (L5-S1) spondylolisthesis and lumbar radiculopathy.

Methods: After Institutional Review Board Approval, charts from 21 consecutive patients with L4-L5 or L5-S1 spondylolisthesis and complaints of lower back and radicular pain who underwent endoscopic procedures between 2007 and 2012 were reviewed.

Results: The average pain relief one year postoperatively was reported to be $71.9 \%$, good results as defined by MacNab. The average pre-operative VAS score was 8.48 , indicated in our questionnaire as severe and constant pain. The average one year postoperative VAS score was 2.30 , indicated in our questionnaire as mild and intermittent pain.

Limitations: This is a retrospective study and only offers one year follow-up data for patients with spondylolisthesis undergoing endoscopic spine surgery for treatment of lumbar radiculopathy.

Conclusions: Endoscopic discectomy is a safe and effective alternative to open back surgery. The one year follow-up data presented here appears to indicate that an ultra-minimally invasive approach to the treatment of lumbar radiculopathy in the setting of spondylolisthesis that has a low complication rate, avoids general anesthesia, and is outpatient might be worth studying in a prospective, longer term way.

IRB approval: Meridian Health: IRB Study \# 201206071J

Key words: Endoscopic discectomy, minimally-invasive, transforaminal, spondylolisthesis

Pain Physician 2014; 17:E703-E708 umbar degenerative spondylolisthesis is a common entity (incidence between $4 \%$ and $8 \%$ ) that occurs mainly in elderly patients. Degenerative lumbar spondylolisthesis is the slipping of one vertebra past its normal alignment to its adjacent vertebra as a result of the degenerative wear of the facet joint that normally helps keep the 2 vertebrae aligned (1). The resulting misalignment can result in canal and foraminal narrowing. Patients can complain of mechanical back pain, claudication symptoms, and radicular symptoms. The trend in surgery has been to offer decompression with instrumented fusion based 
on patient-based outcome data and the inherent instability of the condition (2). The dilemma essential in the surgical treatment of this condition is how to avoid the increased perioperative risk of instrumented fusion in a group that already has more frequent comorbidities as a result of their increased age.

Transforaminal endoscopic discectomy and foraminotomy is an ultra-minimally invasive outpatient surgical option available to patients that does not require general anesthesia. The authors describe here their experience with treating patients with L4-L5 or L5-S1 spondylolisthesis, who present with persistent lumbar radiculopathy despite conservative non-operative treatment, with endoscopic discectomy and forminotomy. The technique described here utilizes an ultra-minimally invasive endoscopic transforaminal technique in an awake patient utilizing a posterolateral approach for 1) removal of the ventral superior articular process (foraminoplasty), 2) transforaminal intracanal access for removal of a lumbar disc herniation, and 3) reduction of the superior endplate to treat lumbar radicular symptoms that resulted from a spondyloslisthesis. A retrospective study of the average patient pain relief up to one year post-endoscopic discectomy and foraminotomy is presented.

\section{Methods}

\section{Participants}

After Institutional Review Board Approval, charts from 21 consecutive patients with L4-L5 (13 patients) or L5-S1 (8 patients) spondylolisthesis (mean age of 63.4, 6 women [29\%] and 15 men [71\%]) and complaints of lower back and radicular pain who underwent endoscopic procedures between 2007 and 2012 were reviewed.

\section{Transforaminal Endoscopic Discectomy and Foraminotomy}

Patients were selected for treatment based on the results of their magnetic resonance imaging (MRI), physical exam, dermatomal pain pattern, and favorable response to transforaminal injection. All patients considered for endoscopic surgical treatment had already exhausted more conservative treatments which included, but were not limited to, physical therapy and epidural steroid injections. Patients treated had degenerative spondylolisthesis without pars defects and without instability as indicated by movement $<3 \mathrm{~mm}$ on flexion-extention spine $x$-rays. Patients included in the study had at least $3 \mathrm{~mm}$ slippage of one vertebral body over the other, but not more than $25 \%$ slippage (Meyerding Grade I) (Table 1).

Patients were positioned in the lateral decubitus position with the operating room table reversed and the flank over the break in the table. A roll was placed under the flank and the table flexed to open the disc space. Anesthesia consisted of mild sedation using versed and fentanyl and $1 \%$ lidocaine local anesthetic. The level of anesthetic was titrated so the patient was able to communicate with the surgeon throughout the procedure. The authors feel that this is the safest way to prevent nerve injury.

The Joimax TESSYS endoscopic system was used for the procedure. Percutaneous entry was established entering through the skin $10-16 \mathrm{~cm}$ lateral to the midline. Using intermittent fluoroscopic guidance, alternating between tunnel view (bull's eye), lateral and anterior-posterior (AP) view a $25 \mathrm{~cm} 18$ gauge needle was advanced and placed in the disc space through Kambin's triangle, between the exiting and traversing nerves. An AP fluoroscopic view was used so the disc space was not entered before the needle was past medial border of the pedicle. This ensured that the needle was not in the central spinal canal, avoiding the dural sac. A guidewire and curved dilator were used to negotiate the superior endplate or any bone spurs in the trajectory of the endoscope. The approach is essentially a Seldinger approach using a flexible wire and small curved dilator to negotiate the obstructed foramen so that sequential reamers can next be used to enlarge the foramen by removing the ventral aspect of the superior articular process (SAP) and endplate. The beveled working cannula, $8.0 \mathrm{~mm}$ in outer diameter, was then placed over the sequential dilators. Rotating the beveled cannula and endoscope allowed for 360 degree visualization of the annulus and exiting and traversing nerve roots. The endoscope used had an optical angle of 30 degrees. When the scope is docked in the neural foramen, the anatomical view is that of Kambin's triangle. The epidural space, traversing nerve root, and residual SAP are visualized above the horizon line of the scope and the disc pathology below the horizon line. The beveled end of the working cannula was also used as a nerve root retractor.

The herniation is then located under direct visualization protruding from the annulus of the disc. Portions of the herniation are removed with forceps graspers until the neural foramen is open enough for structures to be identified clearly. The bipolar radiofrequency 
Transforaminal Endoscopic Discectomy with Foraminoplasty

Table 1. Comparisons for determining statistical significance were performed using a Student t-test.

\begin{tabular}{|c|c|c|c|c|c|c|c|c|}
\hline Age & Gender & Level & Side & Pain Type & $\begin{array}{c}\text { Pre-0p } \\
\text { Pain }\end{array}$ & $\begin{array}{c}\text { One Year } \\
\text { Pain }\end{array}$ & $\begin{array}{c}\text { \% Relief } \\
\text { at One } \\
\text { year }\end{array}$ & $\begin{array}{c}\text { Complication } \\
\text { Post-op }\end{array}$ \\
\hline 70 & $\mathrm{~F}$ & L4-L5 & Left & numbness & 90 & 10 & 80 & \\
\hline 61 & M & L5-S1 & Left & sharp, shooting, tingling & 70 & 0 & 100 & \\
\hline 41 & M & L4-L5 & Left & burning, numbness & 70 & 0 & 90 & Reherniation \\
\hline 60 & M & L4-L5 & Right & $\begin{array}{l}\text { shooting, stabbing, dull } \\
\text { ache }\end{array}$ & 70 & 20 & 60 & \\
\hline 72 & M & L5-S1 & Right & crushing, numbness & 100 & 0 & 100 & \\
\hline 67 & M & L5-S1 & Right & sharp, shooting, stabbing & 100 & 35 & 65 & \\
\hline 54 & M & L4-L5 & Right & cutting, stabbing, burning & 70 & 10 & 45 & \\
\hline 78 & M & L4-L5 & Right & crushing & 60 & 0 & 90 & \\
\hline 48 & M & L5-S1 & Right & sharp, pulsing, nagging & 90 & 10 & 80 & \\
\hline 71 & $\mathrm{~F}$ & L5-S1 & Left & $\begin{array}{l}\text { burning, tingling, } \\
\text { numbness }\end{array}$ & 90 & 20 & 80 & \\
\hline 65 & $\mathrm{~F}$ & L5-S1 & Left & sharp, stabbing, shooting & 90 & 90 & 0 & \\
\hline 48 & $\mathrm{~F}$ & L5-S1 & Right & sharp & 100 & 10 & 90 & \\
\hline 71 & M & L4-L5 & Right & dull ache, numbness & 100 & 10 & 90 & \\
\hline 62 & M & L4-L5 & Left & weakness, numbness & 40 & 20 & 50 & \\
\hline 65 & $\mathrm{~F}$ & L4-L5 & Right & sharp, nagging, pressure & 100 & 50 & 50 & \\
\hline 69 & M & L4-L5 & Right & sharp, tingling, throbbing & 90 & 0 & 100 & \\
\hline 75 & M & L5-S1 & Left & shooting, numbness & 100 & 100 & 0 & \\
\hline 52 & M & L4-L5 & Left & $\begin{array}{l}\text { dull ache, nagging, } \\
\text { cramping }\end{array}$ & 80 & 16 & 80 & Reherniation \\
\hline 65 & M & L4-L5 & Right & sharp, burning, nagging & 80 & 10 & 87.5 & Reherniation \\
\hline 73 & $\mathrm{~F}$ & L4-L5 & Left & sharp & 100 & 70 & 30 & \\
\hline 65 & M & L4-L5 & Right & sharp, dull ache & 90 & 0 & 100 & \\
\hline
\end{tabular}

wand is used for further blunt dissection and hemostasis. Partial flavectomy and facetectomy are performed under endoscopic visualization using the trephine instruments and kerrison rongeur. The trephine is used to remove overriding SAP that impinges the foramen, and once removed, the kerrison punch is used to bite and remove the ligamentum flavum thereby exposing the decompressed nerve root. Using the Joimax Shrill@ shaver-drill system, an additional several millimeters of bone can be removed from the base to the apex of the SAP, allowing the remaining hypertrophied ligamentum flavum contributing to stenosis to be visualized and then removed using the biting forceps, kerrisons, and radiofrequency. The area is decompressed and the exiting and traversing nerve roots are identified under direct visualization until noted to be free of impingement from disc and facet. Additional work can be done to excise a portion of the superior endplate of the inferior vertebra contributing to the spondylolisthesis; anterolisthesis and hypertrophy of the inferior endplate can contribute to the stenosis around the nerve root. The endoscopic trephine can be used to shave several millimeters of the posterior vertebral component under direct vision until it appears to be no longer occupying space in the neuroforamen and the traversing and exiting nerve roots are then visualized to be decompressed and floating freely.

Fig. 1 shows a typical sequence of images encountered in the treatment of an L4-L5 spondylolisthsis. Top left is the pre-operative MRI showing the spondylolisthesis at L4-L5 and the disc protrusion and retropulsed superior endplate of $\mathrm{L} 5$. The top 3 fluouroscopic images depict, first, needle entry, second, trephination of the superior articular process and foraminoplasty, and 
third, placement of the beveled working cannula. Bottom left shows the initial image often seen in spondylolisthesis cases: a large overriding SAP and hypertrophied ligamentum flavum. The bottom middle image shows clearly the decompressed traversing L5 nerve root after endoscopic drilling of the SAP has been performed and the ligament has been reduced with the endoscopic kerrison punches. Bottom right is a photograph of the $4 \mathrm{~cm}$ disc fragment removed.

\section{Measures}

Follow-up sheets were filled out by the patients with each visit indicating the location, severity, and duration of pain. Patients were asked to rate their pain using a $0-10$ scale, a modified form of the Visual Analog Scale (VAS). Each patient had MRI confirmation of disc herniation or protrusion prior to the procedure. The overall pain relief in patients was calculated as a percentage of improvement between the preoperative and the one year postoperative VAS score. Overall success rate was then calculated on each of the 21 patients. MacNab criteria was applied to each patient by characterizing pain relief of $75 \%-100 \%$ as excellent, $50 \%-74 \%$ as good, $25 \%-49 \%$ as fair, and $0 \%-24 \%$ as poor (3). Success is based on an excellent, good, or fair outcome (3).

\section{Results}

Of the 21 patients with spondylolisthesis and radiculopathy undergoing endoscopic discectomy and foraminotomy procedures, the average pre-operative VAS score was 8.48 , indicated in our questionnaire as severe and constant pain. The average one year postoperative VAS score was 2.30, indicated in our questionnaire as mild and intermittent pain. A Student t-test confirmed the statistical difference $(P<0.5)$. Independent from the above calculations, each patient is evaluated for overall success based on the MacNab

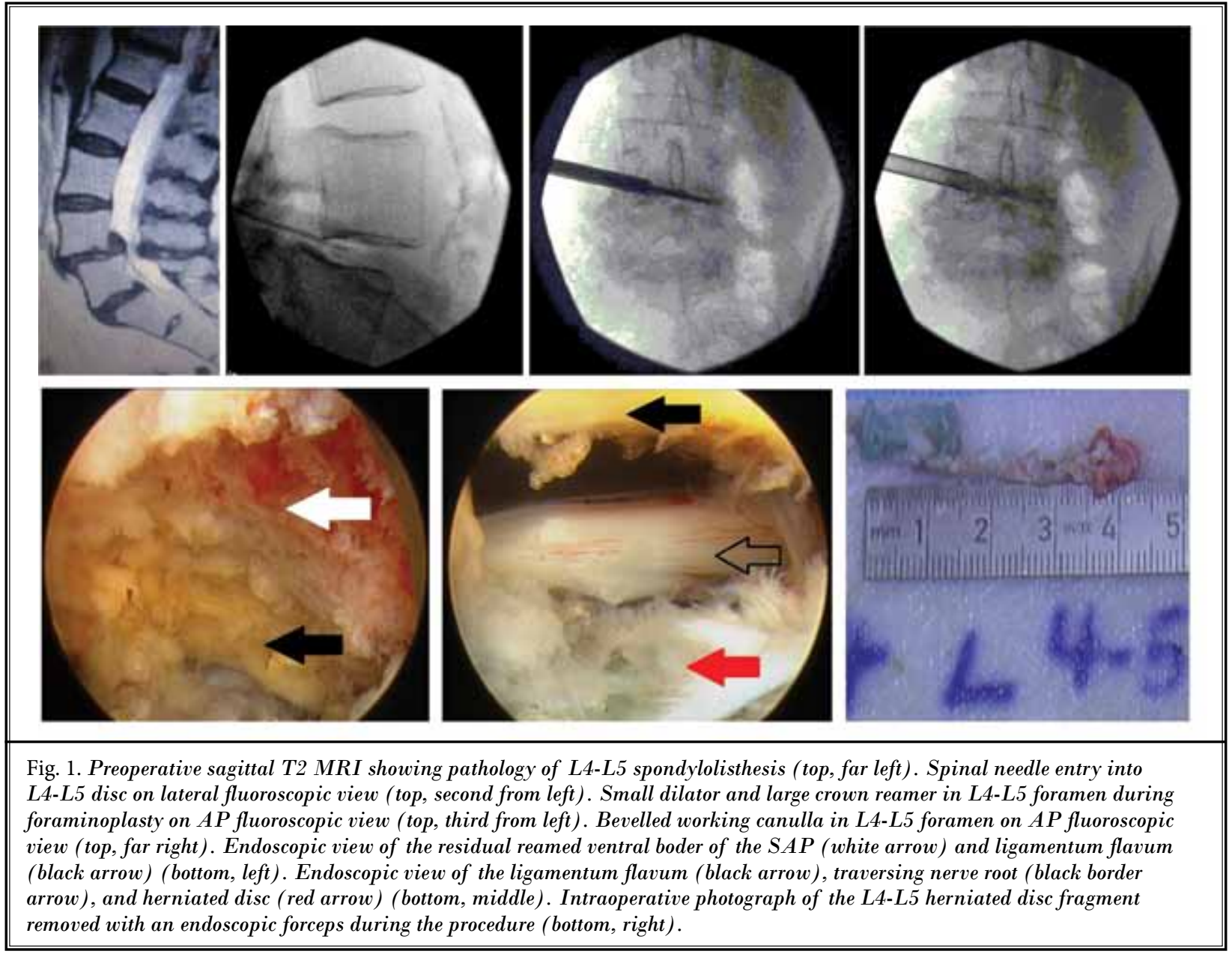


criteria. After one year, excellent results were seen in $13(62 \%)$ patients, good results in $4(19 \%)$ patients, fair results in 2 (9.5\%) patients, and poor results in 2 (9.5\%) patients (Fig. 2). Successful outcomes were seen at one year in about $90 \%$ of patients and at least "good" outcomes in $81 \%$ of patients. The only outcome measure studied here was a measurement of pain. No other functional measures, including walking and claudication measures, were collected or investigated.

There were no reports of infection, dural tear, thrombophlebitis, spinal instability, or vascular injury. There were no serious complications such as cauda equina syndrome or nerve damage resulting in paralysis. Three patients early in the series required a subsequent endoscopic discectomy and foraminotomy on the same side and at the same level in the first 3 month postoperative period to treat symptom recurrence. This was thought to be due to learning curve in the procedure. Second surgeries entailed more aggressive foramiotomies and removal of the inferior endplate. The follow-up for these patients are reported as the one year follow-ups after the final operation. The complication rate was $0 \%$ and the repeat surgery rate was $14 \%$. Two patients $(9.5 \%)$ had an unchanged average VAS scores signifying no pain relief. There were no issues with postoperative instability during this one year follow-up, but surveillance flexion-extension $x$-rays were not performed. Previously reported complications can include infection, dysesthesia, thrombophlebitis, dural tear, vascular injury, and death (4).

\section{Discussion}

Patients presenting with degenerative spondylolisthesis can present with mechanical back pain, radicular pain, and symptoms referable to lumbar canal stenosis. The intellectual argument behind fusing is that fusions are used to increase stability and prevent progression of the condition. However it has been shown that spondylolisthesis rarely progresses in adults and is thus more threatening in appearance than in vivo (5). One treatment study of spondylolisthesis using lumbar posterolateral fusion and transpedicular screw fixation showed that although $90 \%$ of patients had stable fusions, $40 \%$ were considered to have poor outcomes due to residual pain (6). It has also been shown that the hardware used in spinal fusion procedures introduces a significant degree of increased stress at that adjacent segment leading to increased hypertrophic degenerative arthritis of the facet joints, spinal stenosis, severe disc degeneration, and spondylolysis acquisita, all of

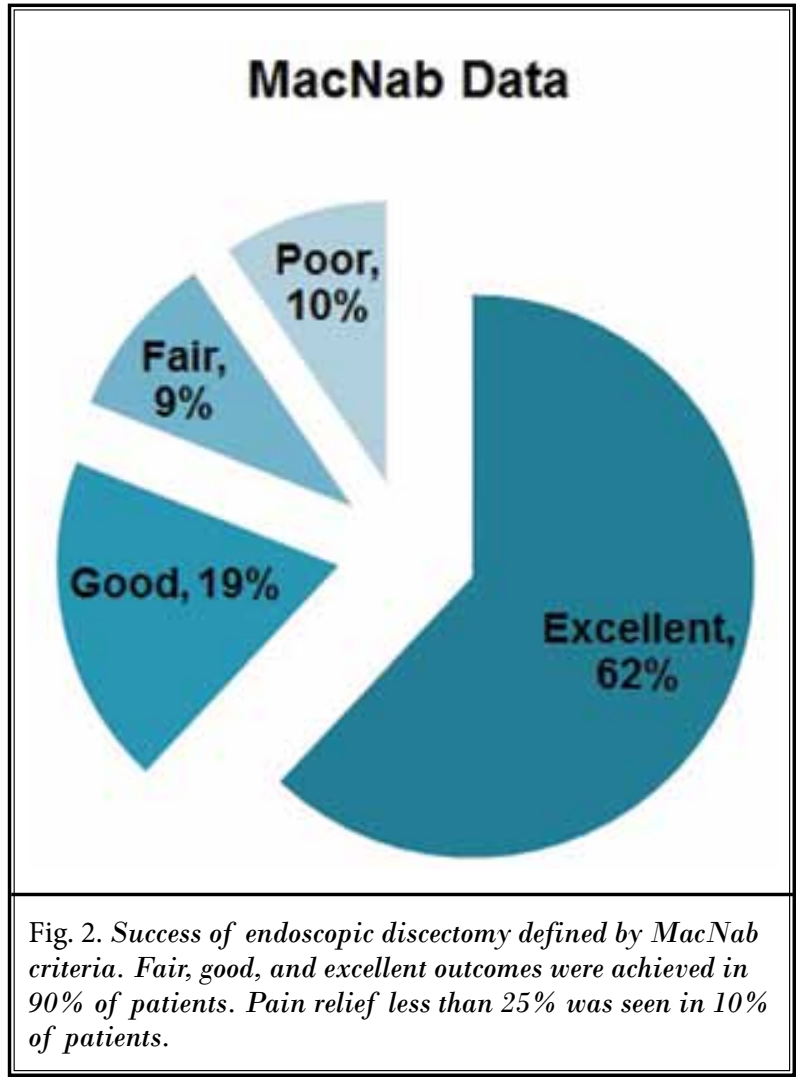

which contribute to lower back and radicular pain (7).

A patient with a symptomatic mobile spondylolisthesis would likely benefit from a decompression supplemented by an instrumented lumbar fusion. A patient with a non-mobile spondylolisthesis who has a decompression without fusion may go on to suffer from the destabilizing effect of the lumbar laminectomy and medial facetectomy. Both cases argue for the addition of fusion to the paradigm for the surgical treatment of spondylolisthesis.

Public demand and technical innovation both fuel advances in more minimally invasive approaches in every surgical field. The authors presenting here have previously published an article as a technical guide to endoscopic tranforaminal surgery in the setting of a grade II spondylolisthesis (8) as well as their results for transforaminal endoscopic surgery for patients with multilevel lumbar degenerative disc disease (9), for geriatric patients (10), and for patients with intracanal pathology (11). Transforaminal endoscopic foraminotomy and discectomy is a treatment option that could be considered for patients who suffer radicular symptoms that result from the narrowing of the foramen that occurs 
in spondylolisthesis. The results presented here for pain relief are comparable to other "cutting edge" minimally invasive surgical approaches to spondylolisthesis that include minimally invasive transforminal lumbar interbody fusion procedures (12-14) and minimally invasive spinous process splitting (15). The procedure described here could be considered for patients with nonmobile spondylolisthesis and radicular symptoms. The procedure focuses on the anatomic pathologies responsible for nerve root compression in spondylolisthesis: shingling of the SAP, protrusion of the disc, and retropulsion of the superior endplate into the inferior aspect of the nerve. The endpoint of the procedure is a 360 degree foraminoplasty: the ventral portion of the SAP is reamed and drilled, the protruding disc is removed with graspers, and the retropulsed superior endplate is reduced with a combination of drilling and grasping.

An awake outpatient endoscopic procedure would be a highly desirable treatment alternative for most elderly patients considering surgery for symptoms associated with degenerative spondylolisthesis. A longer term prospective study is planned to evaluate the length of treatment benefit in this group of patients, as well as provide outcomes data using VAS scores, Oswestry Low Back Pain Disability questionnaire, Beck Depression Inventory, and Medical Outcomes Survey 36-Item Short-Form Health Survey (SF-36).

\section{Conclusion}

Endoscopic surgery for complicated lumbar degenerative disease is not proposed as a cure or solution to the degenerative spine but as a palatable remedy for the patient who wants some improvement without going through a surgery that requires general anesthesia, an inpatient hospital stay, and the possible morbidities associated with an instrumented fusion procedure. Endoscopic discectomy is proposed as a safe and effective alternative to open a lumbar decompression and instrumented fusion for the radicular symptoms that can result in degenerative spondylolisthesis. As the elderly demographic increases in number and gets older, spine physicians need to consider treatment paradigms that factor in risk, patient down-time, and health care costs, and that are specifically tailored to this older population.

\section{References}

1. Jacobsen S, Sonne-Holm S, Rovsing H, Monrad H, Bebuhr P. Degnerative lumbar spondylolisthesis: An epidemiological perspective: The Copenhagen Osteoarthritis Study. Spine 2007; 32:120-125.

2. Kleinstuek FS, Fekete TF, Mannion AF, Grob D, Porchet F, Mutter U, Jeszenszky D. To fuse or not to fuse in lumbar degenerative spondylolisthesis: Do baseline symptoms help provide the answer? Eur Spine J 2012; 21:268-275.

3. MacNab I. Negative disc exploration. JBJS (Am) 1971; 53A:891-903.

4. Yeung AT, Tsou PM. Posterolateral endoscopic excision for lumbar disc herniation. Spine 2002; 22:722-731.

5. Fredrickson BE, Baker D, McHolich WJ, Yuan HA, Lubicky JP. The natural history of spondylosis and spondylolisthesis. JBJS (Am) 1984; 66A:699-707.

6. Schnee CL, Freese A, Ansell LV. Outcome analysis for adults with spondy- lolisthesis treated with posterolateral fusion and transpedicular screw fixation. J Neurosurg 1997; 86:56-63.

7. Lee CK. Accelerated degeneration of the segment adjacent to a lumbar fusion. Spine 1988; 13.3:375-377.

8. Jasper GP, Francisco GM, Aghion D, Telfeian AE. Technical considerations in transforaminal endoscopic discectomy with foraminoplasty for the treatment of spondylolisthesis: Case report. Clin Neurol Neurosurg 2014; 119:84-87.

9. Jasper GP, Francisco GM, Telfeian AE. Clinical success of transforaminal endoscopic discectomy with foraminotomy: A retrospective evaluation. Clin Neurol Neurosurg 2013; 115:1961-1965.

10. Jasper GP, Francisco GM, Telfeian AE. A retrospective evaluation of the clinical success of transforaminal endoscopic discectomy with foraminotomy in geriatric patients. Pain Physician 2013; 16:225-229.
11. Jasper GP, Francisco GM, Telfeian AE. Endoscopic transforaminal discectomy for an extruded lumbar disc herniation. Pain Physician 2013; 16: E31-E35

12. Barbagallo GM, Certo F, Sciacca G, Albanese $V$. Bilateral tubular minimally invasive approach for decompression, reduction and fixation in lumbosacral lythic spondylolisthesis. Neurosurg Focus 2013; 35:Video 9.

13. Kimball J, Yew A, Getachew R, Lu DC. Minimally invasive tubular surgery for transforaminal lumbar interbody fusion. Neurosurg Focus 2013; 35:Video 19.

14. Quraishi NA, Rampersaud YR. Minimal access bilateral transforaminal lumbar interbody fusion for high-grade isthmic spondylolisthesis. Eur Spine ] 2013; 22:1707-1713

15. Nomura $\mathrm{H}$, Yanagisawa $\mathrm{Y}$, Arima J, Oga M. Clinical outcome of microscopic lumbar spinous process-splitting laminectomy. J Neurosurg Spine 2014; 30:1-8. 